\title{
The Contribution of Informatics to Overcoming the Covid-19 Fake News Outbreak by Learning to Navigate the Infodemic
}

\author{
Marianna ISAAKIDOU ${ }^{\mathrm{a}, 1}$ and Marianna DIOMIDOUS ${ }^{\mathrm{a}}$ \\ ${ }^{a}$ Faculty of Nursing, National and Kapodistrian University of Athens, Athens, Greece
}

\begin{abstract}
Daily, people are being exposed to an enormous amount of Covid-19 information, but not all of it is reliable. This phenomenon seriously affects the public health policy effectiveness, because there is a lot of misleading or inaccurate information, which is spreading rapidly and makes it more difficult to restrict the pandemic. Healthcare informatics has emerged as a diverse and important new field. Healthcare informatics applications are becoming more and more popular and are providing easy access to new sources of knowledge. This way, the quality of patient care will improve and productivity will increase. However, people should also learn how to navigate this infodemic properly.
\end{abstract}

Keywords. Healthcare Informatics, fake news, misinformation, Covid-19

\section{Introduction}

The World Health Organisation (WHO) has declared that false allegations are spreading faster than the virus itself and has already defined the situation as a "global information pandemic" [1]. The word "Infodemic" is consisted of the words "information" and "epidemic" or "pandemic", which means "epidemic/pandemic of information" [2]. Due to Covid-19, another word has been added to people's vocabulary daily, called: "epidemiology", which is the branch of medical science that deals with how diseases are transmitted and can be controlled in populations [1]. So, during the current pandemic, a new word has been aroused, called: "infodemiology". The term "Infodemiology" is defined as "the science of distribution and determinants of information in an electronic medium, specifically the Internet or in a population, with the ultimate aim to inform public health and public policy" [3].

The great amount and multimodality of data produced in health, give a key role of data analytics methods as a speciality of health informatics. As a result, an increased interest appeared in development of Machine Learning. Furthermore, techniques with their foundations in artificial neural networks, like Deep Learning, have emerged in recent years as a powerful tool and promise to reshape the future of Artificial

${ }^{1}$ Corresponding Author, Marianna Isaakidou, PhD cand. Faculty of Nursing, School of Health Sciences, National and Kapodistrian University of Athens, Athens, Greece; E-mail: marianis@nurs.uoa.gr. 
Intelligence. Newly appeared online platforms are already operating to reduce the effects of fake news [4].

\section{Methods}

A systematic review of academic literature was conducted, aiming to identify strategies in tackling online misinformation and fake news about public health and especially the Covid-19 pandemic. Other articles were reviewed, involving vaccine misinformation, as well as online and open provided information by health-related authorities, regarding the phenomenon of fake news in the healthcare sector. A systematic review of academic literature was performed using mainly PubMed and ScienceDirect databases in July and August 2021 using terms like "infodemic", "healthcare informatics", "fake news", "Covid-19". In total, 58 academic publications identified and extracted for further assessment. Other than the title, abstract and full-text assessment, 16 articles were selected for inclusion in this literature review. In addition to the systematic review of academic literature, research has also been contacted on National Websites dealing with Public Health and an overview of where people can find accurate and proper information on health issues is presented. This article also attempts to report the sources of Covid-19 misinformation and to find ways to navigate with safety the various websites dealing with information on the pandemic.

\section{Results}

The Covid-19 infodemic is full of false claims, half-backed conspiracy theories and pseudoscientific therapies, regarding the diagnosis, treatment, prevention, origin, and spread of the virus. Reliable and authorised "places" to search for accurate worldwide data about Covid-19 infodemic and all useful information needed, are the websites of every national Ministry of Health and any other national public health authorities, as well as other global organisations, such as the World Health Organisation (WHO), the European Centre for Disease Prevention and Control (ECDC) website, and the Centre for Disease Prevention and Control (CDC) website. It is worth mentioning that as we learn more about the virus, information may change or vary $[5,6]$.

Worldwide data sources for Covid-19 and healthcare issues related to it (e.g. Covid-19 vaccines, behaviours against them, etc) are already starting to increase and healthcare informatics applications are becoming a very useful tool of defence [7]. Previous works proved that "Machine Learning" application seems very useful in recognising and striking out fake news and other disinformation $[8,9,10]$.

The WHO has developed guidelines to help individuals, community leaders, governments and the private sector understand some key actions they can take to manage the Covid-19 infodemic. A very important intervention by the WHO is working closely with more than 50 digital companies and social media platforms, including the most popular ones such as Facebook, Twitter, Viber, and others, to ensure that science-based health messages from the organisation itself or other official sources appear first when people search for health information. The WHO has also collaborated with the Government of the United Kingdom on a digital campaign to raise awareness on misinformation concerning the coronavirus and to encourage individuals to report false or misleading online content [1]. Another awareness campaign by the WHO 
collaborated with the UK is called: "Stop The Spread", which clearly urged people to double-check all information. An additional effort to limit the infodemic was the campaign called "Reporting Misinformation", which urged people to not only verify information, but also report misinformation in various social media platforms. The WHO, also, created an innovative online game called "Go Viral!". Research has shown that even one game can reduce perceived reliability of fake news by an average of $21 \%$ [11]. Lastly, the WHO has created the "EPI-WIN", its own Information Network for Epidemics that seeks to give everyone access to accurate and valid advice or information from trusted sources on public health emergencies [12].

The ECDC website is also ensuring a maximum level of transparency. It screens up to 500 sources to collect Covid-19 figures from 196 countries. It screens official social media accounts (e.g. Facebook) from national authorities. Social media sources are screened to gather additional information, which can be validated by the official sources previously mentioned. This process is being refined constantly, as to ensure the validity and reliability of the data and it is very useful for monitoring and interpreting the dynamics of the Covid-19 pandemic not only in the EU, but also worldwide [13].

The CDC website is also exploring and monitoring similar activities to support the global effort against SARS-CoV-2. The "COVID Data Tracker" finds maps and tracks cases, deaths, and trends of COVID-19 in the United States and it is being updated daily [14]. Additionally, the CDC Health Alert Network (HAN) updates a huge number of guidelines and recommendations on how to evaluate and identify patients who should be tested for Covid-19. The CDC has, also, updated travel guidance to closely monitor and respond to the Covid-19 outbreak [15].

\section{Discussion}

People don't usually give it a second thought before sharing information on social media and forget to verify if it is fake or true. It is extremely useful to teach people how to identify misinformation and how to navigate this infodemic. First of all, they have to assess the source and find out who shared the information and where they got it from. Attractive and catchy headlines are being used to mislead people intentionally. Therefore, people should read the entire article carefully and not just the headline. In addition, they should identify the author's name to ensure they are real and credible. It would be extremely helpful if people were to check the date of the article and any other biases. Examination of the supporting evidence with fact-checkers to debunk misinformation could also be enlightening $[1,8]$. There are different ways to identify fake news and stay accurately informed. People can verify fake news either by traditional verification methods (e.g. getting on to the primary source and verifying it) or online verification tools (e.g. Machine Learning).

The pandemic has been the breeding ground for the germination of misinformation, conspiracy theories and, in general, a climate of suspicion and uncertainty in the face of any message about the situation. Through today's different communication platforms, information flows unfiltered, generated by any individual without credentials to report on specialised topics, giving way to the invention of myths and stories that easily go viral, activating waves of opinion and diffuse beliefs that deepen the climate of misinformation and mistrust [6]. Scientists, health professionals, as well as journalists, should take serious steps to help the public identify and recognise fake news. 


\section{Conclusions}

The fake news crisis is putting the public health at risk as it is taking over social media. If we want to win the war against fake news and misinformation, there is an urgent need to train people on the nature of social media and how to use them effectively. However, personal responsibility is the first and fundamental step to protecting our society from the dangerous phenomenon of fake news. Future steps to combat fake news could be to further apply machine-based classification methods on different types of "fake news". The goal is to develop algorithms that make predictions about future instances. For example, an algorithm could be trained with pre-established indicators or features from a training set to predict its corresponding category for unseen instances by a set of learned rules (e.g. Decision Trees) [16]. A new era of AI is inevitable.

\section{References}

[1] WHO (2021), Let's flatten the infodemic curve. Accessed in 12 July 2021. Available from: https://www.who.int/news-room/spotlight/let-s-flatten-the-infodemic-curve.

[2] Isaakidou M, Zoulias E, Diomidous M. Investigating the Impact of Misinformation Sources on Health Issues: Implications for Public Health. Stud Health Technol Inform. 2021 May 27;281:494-495. doi: 10.3233/SHTI210211.

[3] Eysenbach G. Infodemiology and Infoveillance: Framework for an Emerging Set of Public Health Informatics Methods to Analyze Search, Communication and Publication Behavior on the Internet. J Med Internet Res 2009;11(1): e11. doi: 10.2196/jmir.1157.

[4] Ravi D, Wong C, Deligianni F, Berthelot M, Andreu-Perez J, Lo B, Yang GZ. Deep Learning for Health Informatics. IEEE J Biomed Health Inform. 2017 Jan;21(1):4-21.

[5] European Centre for Disease Prevention and Control (ECDC). Countering online vaccine misinformation in the EU/EEA. Stockholm: ECDC; 2021, ISBN: 978-92-9498-542-2 doi: 10.2900/329304. Accessed in 23 July 2021. https:/www.ecdc.europa.eu/sites/default/files/documents/ Countering-online-vaccine-misinformation-in-the-EU-EEA.pdf.

[6] Bin Naeem S, Bhatti R, and Khan A. An exploration of how fake news is taking over social media and putting public health at risk. Health Info Libr J. 2021 Jun;38(2):143-149. doi: 10.1111/hir.12320.

[7] Darvish A, Bahramnezhad F, Keyhanian S, Navidhamidi M. The Role of Nursing Informatics on Promoting Quality of Health Care and the Need for Appropriate Education. Glob J Health Sci. 2014 Jun 25; 6(6):11-8. doi: 10.5539/gjhs.v6n6p11.

[8] Isaakidou M, Zoulias E, Diomidous M. Machine Learning to Identify Fake News for COVID-19. Stud Health Technol Inform. 2021 May 27;281:108-112. doi: 10.3233/SHTI210130.

[9] Raju V, Mohd J, Ibrahim HK, Abid H. Artificial Intelligence (AI) applications for COVID-19 pandemic, Diabetes \& Metabolic Syndrome. Clinical Research \& Reviews. 2020;14(4):337-339.

[10] Hunt K, Agarwal P, Zhuang J. Monitoring Misinformation on Twitter During Crisis Events: A Machine Learning Approach. Risk Anal. 2020 Nov 14. doi: 10.1111/risa.13634.

[11] WHO (2021), Fighting misinformation in the time of COVID-19, one click at a time, accessed in 12 Aug 2021, https://www.who.int/news-room/feature-stories/detail/fighting-misinformation-in-the-timeof-covid-19-one-click-at-a-time.

[12] WHO (2021), Risk communication-EPI-WIN: WHO Information Network for Epidemics, accessed in 8 Aug 2021, https://www.who.int/teams/risk-communication.

[13] European Centre for Disease Prevention and Control (2021), How ECDC collects and processes COVID-19 data L, accessed in 24 Aug 2021, https://www.ecdc.europa.eu/en/covid-19/data-collection.

[14] Taveras J, León A, Tejeda F, Infodemic and disinformation of the COVID-19: A reflect of the systemic crisis in the post-truth era, accessed in 29 August 2021, citibeats.com/infodemic-and-disinformation-ofthe-covid-19-a-reflect-of-the-systemic-crisis-in-the-post-truth-era/.

[15] Centre for Disease Prevention and Control (CDC). Updated Guidance on Evaluating and Testing Persons for Coronavirus Disease 2019 (COVID-19), accessed in 30 Aug 2021, https://emergency.cdc.gov/han/2020/han00429.asp.

[16] Maria D. Molina, S. Shyam Sundar, Thai Le, Dongwon Lee. "Fake News" Is Not Simply False Information: A Concept Explication and Taxonomy of Online Content. American Behavioral Scientist 2021;65(2):180-212. https://doi.org/10.1177/0002764219878224. 